\title{
Beneficial effects of restrictive annuloplasty on subvalvular geometry in patients with functional mitral regurgitation and advanced cardiomyopathy
}

\author{
Satoshi Kainuma, MD, PhD, ${ }^{\text {a,b }}$ Toshihiro Funatsu, $\mathrm{MD}, \mathrm{PhD},{ }^{\mathrm{a}}$ Haruhiko Kondoh, $\mathrm{MD}, \mathrm{PhD},{ }^{\mathrm{a}}$ \\ Takenori Yokota, MD, PhD, ${ }^{a}$ Shusaku Maeda, MD, ${ }^{a}$ Yasuhiro Shudo, MD, PhD, ${ }^{a, b}$ \\ Hajime Matsue, MD, PhD, ${ }^{\mathrm{a}, \mathrm{b}}$ Masami Nishino, MD, PhD, ${ }^{\mathrm{c}}$ Takashi Daimon, $\mathrm{PhD},{ }^{\mathrm{d}}$ \\ Koichi Toda, MD, PhD, ${ }^{\mathrm{a}, \mathrm{b}}$ Yoshiki Sawa, MD, PhD, ${ }^{\mathrm{b}}$ and Kazuhiro Taniguchi, $\mathrm{MD}, \mathrm{PhD}^{\mathrm{a}}$
}

\section{ABSTRACT}

Objectives: The effects of restrictive mitral annuloplasty (RMA) on subvalvular geometry remains unknown. We evaluated changes in left ventricular (LV) function, severity of mitral regurgitation (MR), and leaflet tethering parameters after RMA and clarified their associations.

Methods: In 44 patients with clinically relevant functional MR who underwent RMA, distances between papillary muscle (PM) tips and anterior mitral annulus (PM tethering distance), leaflet angles relative to lines connecting annuli, and interpapillary muscle distance (IPMD) were serially quantified.

Results: One month after surgery, LV function and MR severity improved with decreased anterior ( $34 \pm 5$ to $30 \pm 4 \mathrm{~mm}$ ) and posterior PM tethering distance ( $37 \pm 4$ to $32 \pm 4 \mathrm{~mm}$ ), anterior leaflet angle ( $32 \pm 8^{\circ}$ to $22 \pm 7^{\circ}$ ), and IPMD ( $31 \pm 6$ to $25 \pm 5 \mathrm{~mm}$ ), whereas these variables remained abnormal and posterior leaflet angle increased ( $34 \pm 8^{\circ}$ to $48 \pm 14^{\circ} ; P<.01$ for all). During follow-up (66 \pm 37 months), these effects were maintained in 33 patients without MR recurrence, whereas 11 with it showed worsened tethering with less LV function recovery. Multiple linear regression analyses identified that change in MR severity from baseline to 12-month examination independently associated with corresponding change in IPMD (parameter estimate of 0.100 with standard error of 0.039; $P=.019$ ) and that in posterior PM tethering distance (parameter estimate of -0.104 with standard error of $0.045 ; P=.035$ ), whereas not with change in posterior-leaflet angle. The IPMD change was independently associated with change in LV end-systolic dimension (parameter estimate of 0.299 with standard error of $0.110 ; P=.013$ ).

Conclusions: The RMA procedure partially relieved leaflet tethering, evidenced by decreased tethering distances and IPMD; the latter was the main determinant of MR. These beneficial effects might be mainly attributed to post-RMA reverse LV remodeling, potentially offsetting the negative effect of augmented posterior leaflet angle in selected patients. (J Thorac Cardiovasc Surg 2018;156:630-8)

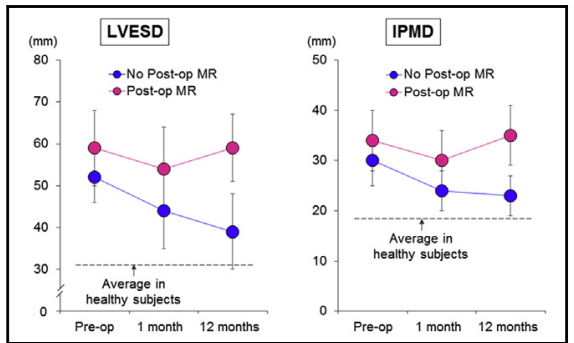

Serial changes in LV end-systolic dimension and IPMD after RMA in patients with functional MR.

\section{Central Message}

In patients with clinically relevant functional MR, the RMA procedure partially relieved leaflet tethering, evidenced by decreased tethering distances and IPMD; the latter was the main determinant of MR.

\section{Perspective}

Substantial improvements in subvalvular geometry might be mainly attributed to post-RMA reverse LV remodeling, potentially offsetting the negative effect of augmented posterior leaflet angle induced by RMA. The finding of the independent association between MR severity and IPMD supports the concept that concomitant PM approximation with RMA could be beneficial for durable repair (valve competence).

See Editorial Commentary page 639.
From the ${ }^{\mathrm{a}}$ Department of Cardiovascular Surgery and ${ }^{\mathrm{c}}$ Department of Cardiology, Japan Organization of Occupational Health and Safety, Osaka Rosai Hospital, Sakai, Osaka, Japan; ${ }^{b}$ Department of Cardiovascular Surgery, Osaka University Graduate School of Medicine, Suita, Osaka, Japan; and ${ }^{\mathrm{d} D e p a r t m e n t}$ of Biostatistics, Hyogo College of Medicine, Nishinomiya, Hyogo, Japan.

This research was partially supported by research funds to promote the hospital function of the Japan Organization of Occupational Health and Safety Osaka Rosai Hospital.
Received for publication June 29, 2017; revisions received Nov 15, 2017; accepted for publication Nov 29, 2017; available ahead of print Feb 1, 2018.

Address for reprints: Kazuhiro Taniguchi, MD, PhD, 1179-3 Nagasone-cho, Kita-ku, Sakai, Osaka 591-8025, Japan (E-mail: kataniguchi1953@gmail.com). $0022-5223 / \$ 36.00$

Copyright (C) 2017 by The American Association for Thoracic Surgery https://doi.org/10.1016/j.jtcvs.2017.11.090 

Abbreviations and Acronyms
IPMD = interpapillary muscle distance
$\mathrm{LV}=$ left ventricular
$\mathrm{MR}=$ mitral regurgitation
$\mathrm{PM}=$ papillary muscle
RMA $=$ restrictive mitral annuloplasty

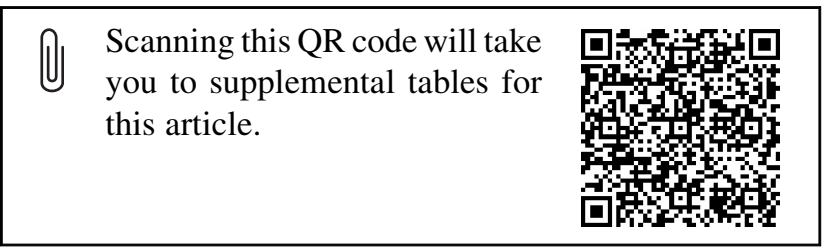

Although the exact mechanism causing persistent or recurrent mitral regurgitation (MR) is still controversial, it has been reported that leaflet tethering by the displaced papillary muscles (PMs) because of regional or global left ventricular (LV) dysfunction has been suggested as the main mechanism of functional MR. ${ }^{1-10}$ In particular, the posterior PM tethering distance (ie, the distance between posterior PM tips and anterior annulus) has been suggested as a major determinant of MR severity. ${ }^{1}$ Several clinical studies have also shown that interpapillary muscle distance (IPMD), as a reliable index of dysfunctional subvalvular apparatus in patients with functional MR, can predict postrepair MR and indicate the need for a procedure complementary to ring annuloplasty. ${ }^{2}$ Of note, it remains unclear to what extent restrictive mitral annuloplasty (RMA) alone relieves subvalvular leaflet tethering, the main mechanism of functional MR. These findings highlight the need for a comprehensive understanding of the geometric changes in the mitral valvular-ventricular complex after RMA to optimize a surgical technique. Therefore, the purpose was to investigate leaflet configurations in patients with functional MR before and after surgical annuloplasty, and to clarify the main determinant contributing to the change in MR severity and that in the relevant subvalvular geometry.

\section{METHODS}

In this study we enrolled patients with clinically relevant functional MR secondary to ischemic or nonischemic advanced cardiomyopathy (defined as severely impaired LV systolic function with an ejection fraction $\leq 40 \%$ ) who underwent RMA using a complete annuloplasty ring between 2004 and 2015. Each had functional MR secondary to LV remodeling and systolic restrictive motion of mitral leaflets in echocardiography findings (ie, Carpentier classification type IIIb). Patients were excluded if they had organic MR, less LV remodeling with an ejection fraction $>40 \%$, or myocardial infarction between preoperative and postoperative echocardiographic assessments. In addition, patients who underwent RMA with partial ring or concomitant surgical ventricular reconstruction were also excluded. Moreover, 12 healthy subjects with no history of cardiovascular disease and with normal Doppler echocardiographic examination were recruited for the control group to accurately evaluate what extent the RMA alone relieves subvalvular leaflet tethering.

All patients underwent RMA through a median sternotomy under a mild hypothermic cardiopulmonary bypass, with antegrade and retrograde intermittent cold blood cardioplegia. No other adjunct procedures were performed on the mitral valve itself. The degree of downsizing was determined not only by the annular geometry of the native valve, but also according to the patients' body surface area. ${ }^{11,12}$ The study protocol was approved by an institutional ethics committee, and all patients provided informed consent before undergoing the procedures.

\section{Echocardiography}

Two-dimensional and Doppler echocardiography procedures were performed by expert echocardiographic examiners 1 week before surgery (baseline), 1 month after surgery, and annually thereafter. Anatomic and Doppler measurements were performed according to the recommendations of the American Society of Echocardiography. The severity of MR was determined by the regurgitant volume and ratio of color Doppler jet area to left atrial area in mid-systole and then classified as none or trivial $(0)$, mild $(1+)$, moderate $(2+)$, moderate to severe $(3+)$, or severe $(4+)$.

\section{Annular and Subvalvular Geometry}

The echocardiographic assessments of annular and subvalvular geometry were previously described in detail (Figure 1 ). ${ }^{3}$ The annular anteroposterior diameter was measured as the distance at the center of the anterior and posterior mitral annulus in the long-axis view at end-systole (Figure 2, A). Tenting height was measured as the perpendicular distance between the coaptation point of the mitral leaflets and the line connecting the annular hinge points to estimate the apical displacement of the mitral leaflets (Figure 2, A). The coaptation length of the anterior and posterior mitral valve leaflet was directly calculated at mid-systole (Figure 2, B). The PM tethering distance was measured from the tip of the anterior and posterior PM to the anterior mitral annulus to estimate lateral and medial PM displacement, respectively (Figure 2, $C$ and $D$ ). The degree of leaflet angle was measured as the angles between each leaflet and the line connecting the annuli (Figure 2,E). The IPMD was measured as the distance between the anterior and posterior PM body in the short-axis views (Figure 2, F).

\section{Follow-up Examinations}

Every 6 months to 1 year, each patient was assessed in the department as well as by their primary cardiologist. Functional status was assessed according to New York Heart Association criteria for symptoms of heart failure. Clinical follow-up examinations were completed for all patients with a mean duration of $66 \pm 37$ months (range, 2.2-138 months).

\section{Statistical Analysis}

Patients were classified into 2 groups depending on the MR severity after RMA. Patients with MR of less than moderate grade at any follow-up examination comprised the no recurrence MR group, whereas those with MR of moderate grade or higher comprised the MR recurrence group Continuous variables are presented as mean $\pm \mathrm{SD}$, and categorical variables as frequencies and proportions. For the continuous variables, comparisons between the 2 groups were made using the Welch $t$ test. For the categorical variables, the 2 groups were compared using Fisher exact test. The echocardiographic variables over time were analyzed using a mixed effects model for repeated measures, including factors for group, time, and interaction between group and time. The variance-covariance matrix of the observation in the linear mixed effects models was assumed to be unstructured. Assessment time points were treated as categorical factors. 


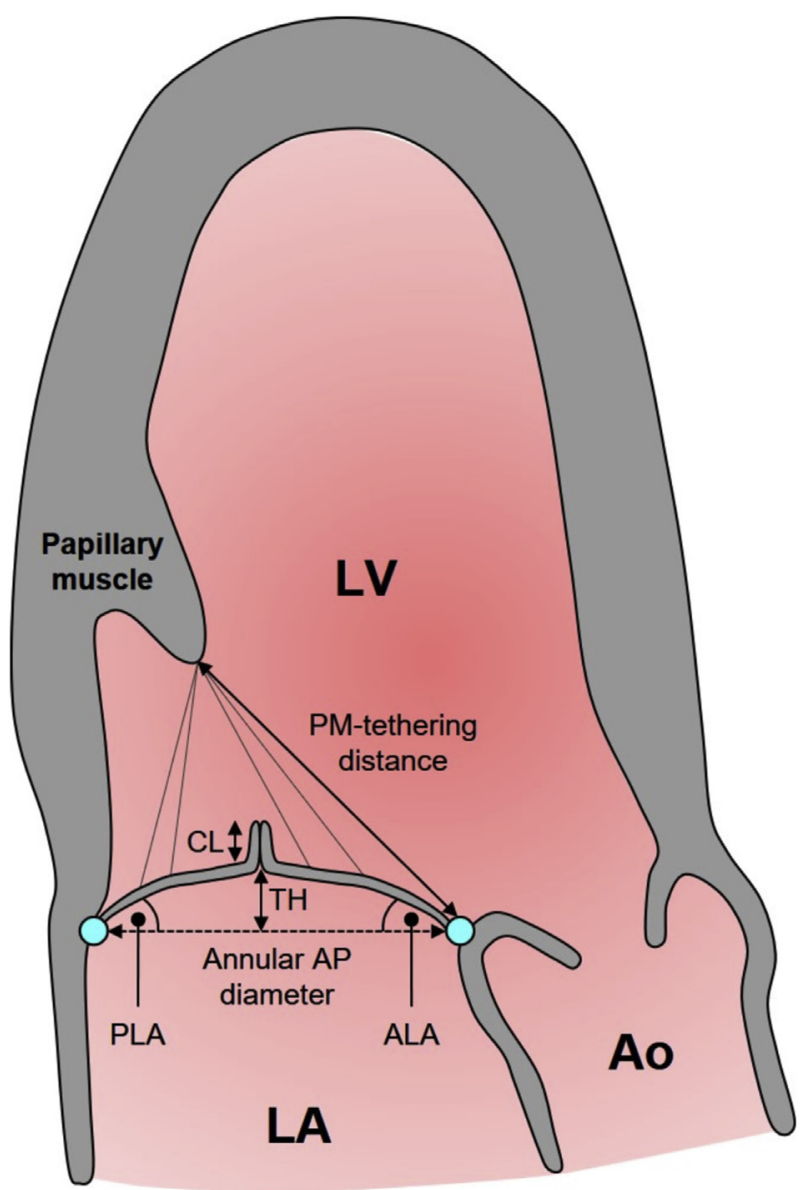

FIGURE 1. Schema of transthoracic echocardiographic assessments of annular and subvalvular geometry. $L V$, Left ventricle; $P M$, papillary muscle; $C L$, coaptation length; $T H$, tenting height; $A P$, anteroposterior; $P L A$, posterior leaflet angle; $A L A$, anterior leaflet angle; $L A$, left atrium; $A o$, aorta.

Multiple linear regression analyses were used to define the independent determinants for change in MR severity and change in IPMD from baseline to 12-month follow-up. For the multiple linear regression analyses, participants with male sex, changes in LV end-systolic dimension, anterior and posterior PM tethering distances, posterior leaflet angle, and IPMD were introduced into a final model on the basis of information from previous literature or clinical knowledge..$^{1-7}$ The results are summarized as parameter estimates, standard error $t$ statistics, and $P$ values. All $P$ values are 2 -sided and values of $P<.05$ were considered to indicate statistical significance. Statistical analyses were performed using JMP 7.0 statistical software (SAS Institute Inc, Cary, NC).

\section{RESULTS}

\section{Patient Demographic Characteristics}

The study population consisted of 44 patients who met the inclusion criteria. The mean age was $65 \pm 8$ years, and there were 33 male and 11 female participants (Table 1). The control subjects had a gender distribution and average body size similar to those of the patients with functional MR.
One month after the surgery, the severity of MR substantially decreased to less than moderate in most patients, whereas 2 patients had persistent moderate MR. During the follow-up, MR severity tended to re-increase, resulting with 2 patients with early postoperative MR and an additional 9 in whom recurrent MR developed. These 11 patients $(25 \%)$ comprised the group with postoperative MR recurrence and the remaining $33(75 \%)$ comprised the group without it. There was no significant difference between the patients with and without MR recurrence in terms of preoperative clinical and surgical data (Table 1).

\section{Determinants of Change in MR Severity and in the Interpapillary Muscle Distance After RMA}

The multiple linear regression analysis identified the change in the IPMD had the most important contribution to change in MR severity (parameter estimate of 0.100 with standard error of $0.039, P=.019$ ), followed by change in the posterior PM tethering distance (parameter estimate of -0.104 with standard error of $0.045 ; P=.035$ ) (Table E1). The multiple linear regression analysis identified change in the LV end-systolic dimension as the only independent contributor to change in the IPMD (parameter estimate of 0.299 with standard error of $0.110 ; P=.013$ ) (Table E2).

\section{Symptoms 1 and 12 Months After RMA}

One month after surgery, most patients presented with class I (no symptoms) or II heart failure symptoms, with no significant difference between patients with and without MR recurrence $(91 \%$ vs $94 \% ; P=.47)$. The proportion of patients with class III or IV heart failure symptoms increased from baseline to the 12-month follow-up in both groups, with a higher percentage of patients with MR recurrence $(18 \%$ vs $55 \% ; P=.02)$.

\section{Changes in LV Function and Mitral Valve Geometry After RMA}

During the follow-up period ( $66 \pm 37$ months), a total of 513 echocardiographic examinations were performed in the 44 patients enrolled in this study, with a mean number of examinations of $12 \pm 4$ (range, 4-20). Of those, 162 echocardiographic examinations were performed to specifically evaluate the annular and subvalvular geometry, with a mean number of examinations of $3.7 \pm 1.7$ (range, 1-8).

Changes in LV function and mitral valve configuration according to the patients with and without MR recurrence are summarized in Table 2 and Figures 3-5. In general, there were significant differences between the control subjects and patients with functional MR regarding the echocardiographic parameters of LV dimensions and function and mitral valve configuration at baseline. One month after surgery, patients who underwent RMA showed significant reverse LV remodeling as well as 

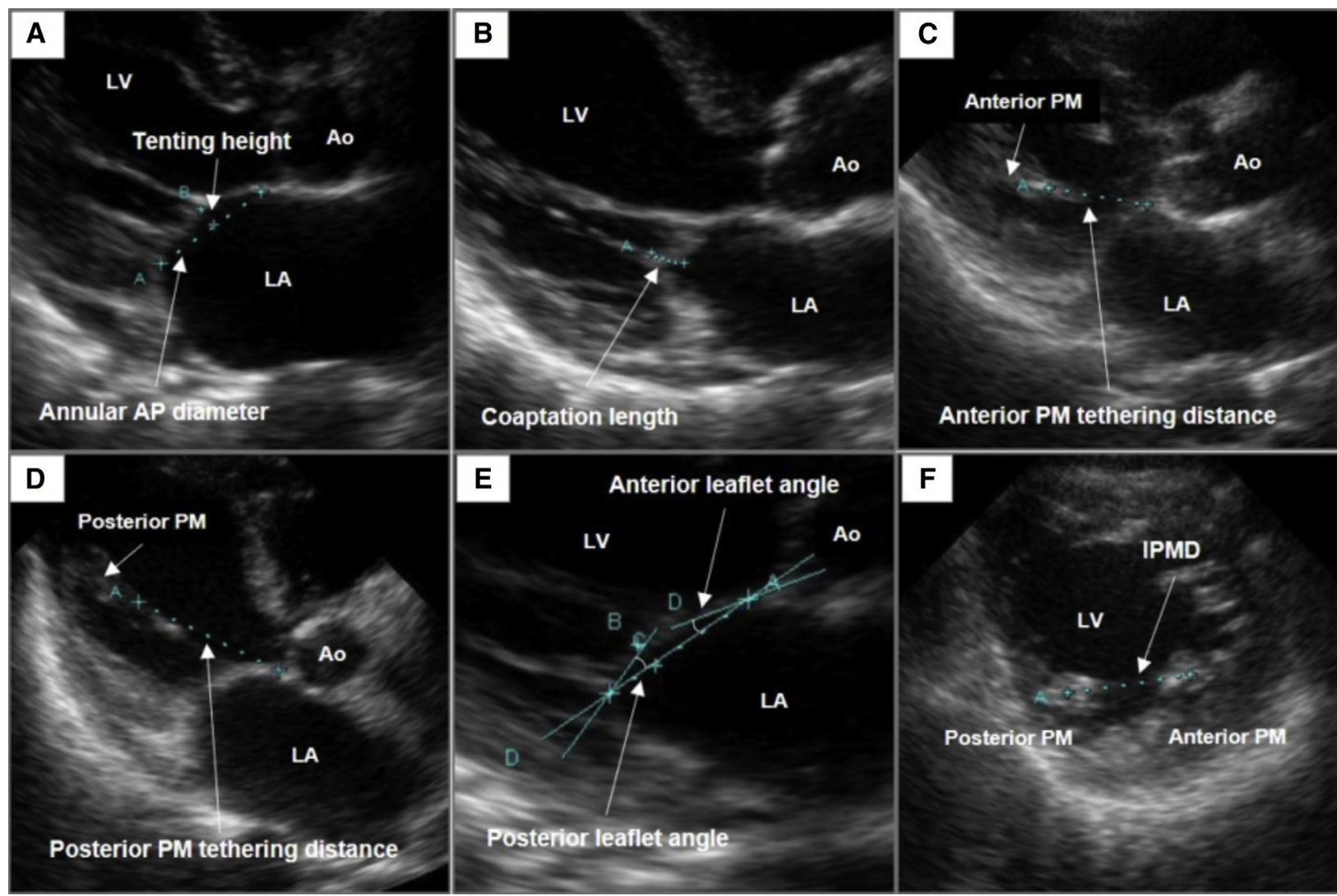

Anterior PM tethering distance

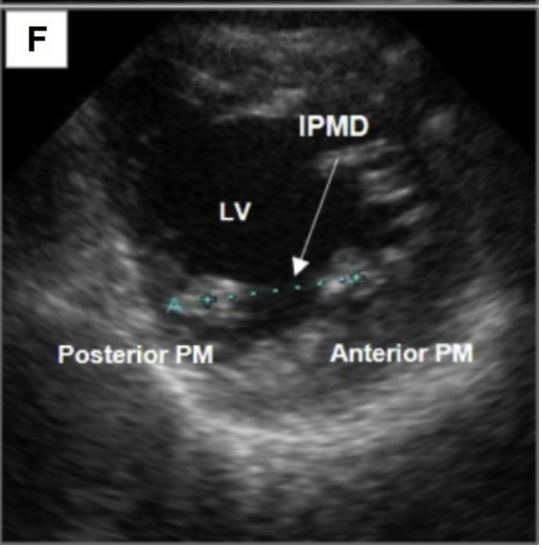

FIGURE 2. Echocardiographic assessments of annular anteroposterior ( $A P$ ) diameter and tenting height (A), coaptation length of mitral leaflets (B), anterior papillary muscle $(P M)$ tethering distance $(\mathrm{C})$, posterior PM tethering distance (D), anterior and posterior leaflet angle (E), and interpapillary muscle distance (IPMD) (F). $L V$, Left ventricle; Ao, aorta; $L A$, left atrium.

annular reduction and relieved leaflet tethering, except for augmented posterior leaflet angle. Thereafter, the parameters of LV function and leaflet tethering showed trends to improve, however some of these values remained abnormal compared with control subjects.

LV function parameters were improved at 1 month after surgery in both groups, with subsequent changes in each group apparently distinctive. Patients without MR recurrence showed further decreases in LV dimensions and improvement in LV ejection fraction afterward, whereas those with MR recurrence showed re-increase in LV dimensions and less improvement in LV ejection fraction (interaction effects $P<.001$ for all), almost returning to the preoperative values (Figure $3, A$ ).

The annular anteroposterior diameter was decreased 1 month after surgery in both groups, which kept constant afterward with no significant differences between patients with and without MR recurrence (time effect $P<.001$; group effect $P=.877$; Figure $3, B$ ). The tenting height was significantly decreased, whereas the coaptation length was increased 1 month after surgery in both groups, indicating relieved apical displacement of the mitral leaflets (time effect $P<.001$ for both; Figures $3, C$ and $4, A$ ). These values in patients without MR recurrence kept constant afterward, eventually reaching almost the same level of the normal subjects. In contrast, these values in patients with MR recurrence were likely to deteriorate thereafter (group effect $P<.001$ for both).

The anterior PM tethering distance and the anterior leaflet angle showed trends to decrease in both groups in a similar manner (time effect $P<.001$ for both; Figure 4, $B$ and $C$ ). In contrast, the posterior leaflet angle increased in both groups 1 month after surgery (time effect $P<.001$; Figure 5, A). Patients without MR recurrence did not show progressive increase in the posterior leaflet angle afterward, whereas those with it showed significant progression (interaction effect $P=.005$ ). The posterior PM tethering distance and IPMD were substantially decreased in both groups (time effect $P<.001$ and .002 , respectively), with subsequent changes in each group apparently distinctive (interaction effects $P=.003$ for both; Figure 5, $B$ and $C$ ). Patients without MR recurrence showed further decreases afterward, whereas those with MR recurrence showed re-increase in these parameters, almost returning to the preoperative values. Consequently, patients without MR recurrence showed symmetrical tethering in 
TABLE 1. Preoperative clinical and surgical data

\begin{tabular}{|c|c|c|c|c|c|}
\hline Variable & Control $(\mathbf{n}=12)$ & All subjects $(n=44)$ & No post-op MR $(\mathbf{n}=33)$ & Post-op MR $(\mathrm{n}=11)$ & $P$ value* \\
\hline Age, y & $33 \pm 6$ & $65 \pm 8$ & $64 \pm 9$ & $67 \pm 5$ & .381 \\
\hline Male, n (\%) & $9(75 \%)$ & $33(75 \%)$ & $25(76 \%)$ & $8(73 \%)$ & .999 \\
\hline Body surface area, $\mathrm{m}^{2}$ & $1.7 \pm 0.2$ & $1.6 \pm 0.2$ & $1.7 \pm 0.2$ & $1.6 \pm 0.2$ & .304 \\
\hline NYHA class III or IV, n (\%) & $0(0 \%)$ & $37(84 \%)$ & $28(85 \%)$ & $9(82 \%)$ & .932 \\
\hline Ischemic etiology, n (\%) & - & $30(68 \%)$ & $22(67 \%)$ & $8(73 \%)$ & .999 \\
\hline \multicolumn{6}{|l|}{ Surgical procedure } \\
\hline RMA alone, n (\%) & - & $16(36 \%)$ & $12(36 \%)$ & $4(36 \%)$ & .999 \\
\hline RMA with CABG, n (\%) & - & $28(64 \%)$ & $21(64 \%)$ & $7(64 \%)$ & \\
\hline \multicolumn{6}{|l|}{ Size of annuloplasty ring } \\
\hline $24 \mathrm{~mm}, \mathrm{n}(\%)$ & - & $29(66 \%)$ & $23(70 \%)$ & $6(55 \%)$ & .331 \\
\hline $26 \mathrm{~mm}, \mathrm{n}(\%)$ & - & $13(30 \%)$ & $8(24 \%)$ & $5(45 \%)$ & \\
\hline $28 \mathrm{~mm}, \mathrm{n}(\%)$ & - & $2(4.5 \%)$ & $2(6.0 \%)$ & $0(0 \%)$ & \\
\hline \multicolumn{6}{|l|}{ Ring type } \\
\hline CE Physio ring, n (\%) & - & $38(86 \%)$ & $29(88 \%)$ & $9(82 \%)$ & .362 \\
\hline CE Physio II ring, n (\%) & - & $4(9.1 \%)$ & $2(6.0 \%)$ & $2(18 \%)$ & \\
\hline SJM rigid saddle ring, $\mathrm{n}(\%)$ & - & $2(4.5 \%)$ & $2(6.0 \%)$ & $0(0 \%)$ & \\
\hline
\end{tabular}

Post-op, Postoperative; $M R$, mitral regurgitation; NYHA, New York Heart Association; RMA, restrictive mitral annuloplasty; $C A B G$, coronary artery bypass grafting; CE, Carpentier-Edwards; SJM, St Jude Medical. *No MR recurrence versus MR recurrence.CE Physio rings are from Edwards Lifesciences Corp (Irvine, Calif).

terms of comparable degree of anterior and posterior tethering, whereas those with MR recurrence showed asymmetrical tethering with displacements most prominent for the posteromedial PM.

\section{DISCUSSION \\ Major Findings}

The major findings of this study can be summarized as follows. In patients with functional MR who underwent RMA: (1) change in MR severity was independently associated with change in IPMD, although not with change in posterior leaflet angle, (2) change in LV end-systolic dimension was the most important and independent contributor to change in IPMD, (3) the proportion of patients with class III or IV heart failure symptoms at the 12-month follow-up was higher in the MR recurrence group, and (4) patients who did not present significant MR recurrence restored subvalvular geometry with substantial reverse LV remodeling, whereas those who presented MR recurrence failed to achieve those improvements.

Green and colleagues showed, in an ovine model, that annuloplasty produces a markedly impaired motion of a

TABLE 2. Serial echocardiographic data in patients with versus without postoperative MR

\begin{tabular}{|c|c|c|c|c|c|c|c|}
\hline & \multirow[b]{2}{*}{ Control $(n=12)$} & \multicolumn{3}{|c|}{ No post-op MR $(\mathbf{n}=33)$} & \multicolumn{3}{|c|}{ Post-op MR $(n=11)$} \\
\hline & & Pre-op & 1 Month & 12 Months & Pre-op & 1 Month & 12 Months \\
\hline \multicolumn{8}{|l|}{ LV function parameters } \\
\hline LVEDD, mm & $49 \pm 3$ & $64 \pm 6^{*}$ & $56 \pm 7 *$ & $53 \pm 7$ & $67 \pm 9^{*}$ & $63 \pm 9^{*}, \dagger$ & $67 \pm 7^{*}, \dagger$ \\
\hline LVESD, mm & $31 \pm 3$ & $52 \pm 6^{*}$ & $44 \pm 9^{*}$ & $39 \pm 9 *$ & $59 \pm 9^{*}, \dagger$ & $54 \pm 10^{*}, \dagger$ & $59 \pm 8^{*}, \dagger$ \\
\hline LVEF, \% & $70 \pm 4$ & $35 \pm 5^{*}$ & $43 \pm 10^{*}$ & $53 \pm 13^{*}$ & $31 \pm 8^{*}$ & $37 \pm 10^{*}$ & $33 \pm 12^{*}, \dagger$ \\
\hline \multicolumn{8}{|l|}{ Annular geometry } \\
\hline Annular AP diameter, $\mathrm{mm}$ & $26 \pm 4$ & $33 \pm 5^{*}$ & $19 \pm 2 *$ & $18 \pm 2 *$ & $32 \pm 3^{*}$ & $19 \pm 3^{*}$ & $18 \pm 2 *$ \\
\hline \multicolumn{8}{|l|}{ Subvalvular geometry } \\
\hline Tenting height, mm & $4.0 \pm 1.4$ & $7.9 \pm 2.3^{*}$ & $3.8 \pm 1.3$ & $4.2 \pm 1.2$ & $8.5 \pm 1.8^{*}$ & $6.0 \pm 2.0^{*}, \dagger$ & $6.2 \pm 2.1^{*}$ \\
\hline Coaptation length, $\mathrm{mm}$ & $9.3 \pm 1.4$ & $4.8 \pm 1.8^{*}$ & $8.7 \pm 2.1$ & $8.6 \pm 1.3$ & $3.5 \pm 1.5^{*}$ & $7.2 \pm 2.2^{*}$ & $5.9 \pm 1.7^{*}$ \\
\hline APM tethering distance, $\mathrm{mm}$ & $27 \pm 2$ & $34 \pm 6^{*}$ & $30 \pm 4$ & $29 \pm 3$ & $31 \pm 4$ & $28 \pm 3$ & $30 \pm 3$ \\
\hline PPM tethering distance, $\mathrm{mm}$ & $29 \pm 3$ & $36 \pm 4^{*}$ & $32 \pm 4$ & $30 \pm 4$ & $37 \pm 5^{*}$ & $31 \pm 4$ & $35 \pm 4^{*}, \dagger$ \\
\hline Anterior leaflet angle, degree & $17 \pm 5$ & $31 \pm 9 *$ & $21 \pm 6$ & $22 \pm 5^{*}$ & $33 \pm 5^{*}$ & $25 \pm 9^{*}$ & $27 \pm 7 *$ \\
\hline Posterior leaflet angle, degree & $23 \pm 7$ & $33 \pm 7 *$ & $44 \pm 11^{*}$ & $44 \pm 10^{*}$ & $41 \pm 8^{*}, \dagger$ & $69 \pm 9^{*}, \dagger$ & $64 \pm 18^{*}, \dagger$ \\
\hline IPMD, $\mathrm{mm}$ & $19 \pm 3$ & $29 \pm 6^{*}$ & $24 \pm 4^{*}$ & $23 \pm 3^{*}$ & $34 \pm 6^{*}$ & $30 \pm 6^{*}, \dagger$ & $34 \pm 6^{*}, \dagger$ \\
\hline
\end{tabular}

Post-op, Postoperative; $M R$, mitral regurgitation; Pre-op, preoperative; $L V$, left ventricular; $L V E D D$, left ventricular end-diastolic dimension; $L V E S D$, left ventricular end-systolic dimension; $L V E F$, left ventricular ejection fraction; $A P$, anteroposterior; $A P M$, anterior papillary muscle; $P P M$, posterior papillary muscle; $I P M D$, interpapillary muscle distance. ${ }^{*} P<.05$ relative to control (analyzed using Welch $t$ test). $\dagger P<.05$ relative to no post-op MR (analyzed using Welch $t$ test). 


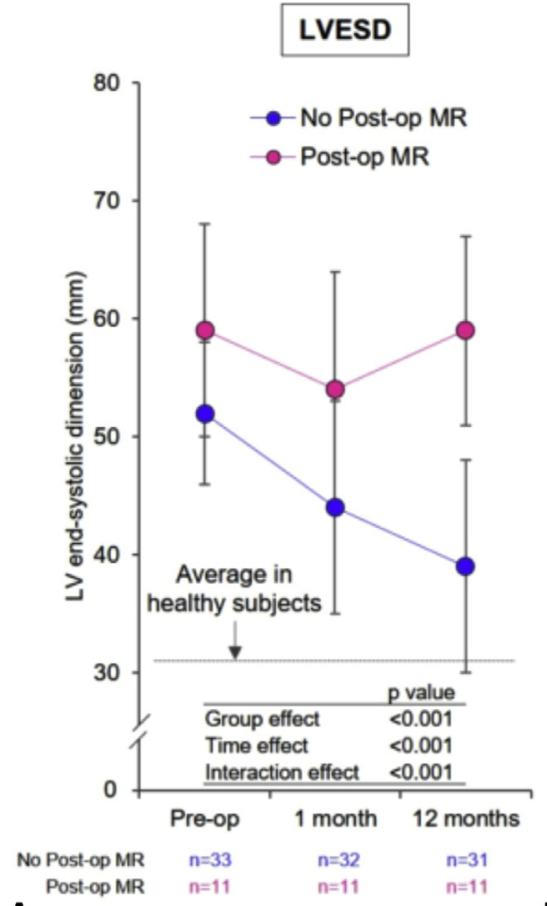

A

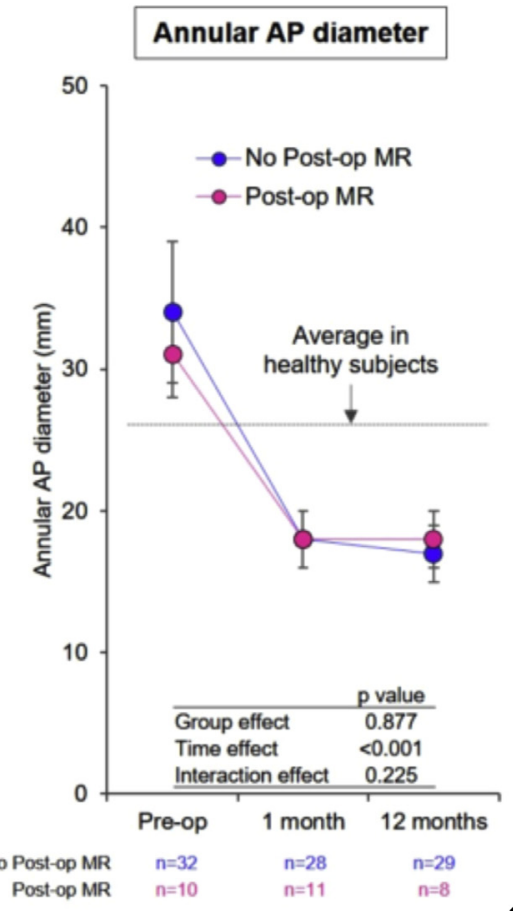

B

FIGURE 3. Serial changes in left ventricle $(L V)$ end-systolic dimension (A), annular anteroposterior $(A P)$ diameter $(\mathrm{B})$, and tenting height $(\mathrm{C})$ according to presence and absence of mitral regurgitation $(M R)$ recurrence. LVESD, Left ventricular end-systolic dimension; Pre-op, preoperative.

"frozen" posterior leaflet, transforming the mitral valve into a unicuspid valve, where the mechanism of closure is performed only by the anterior leaflet. ${ }^{4}$ Kuwahara and colleagues reported that an anterior leaflet longer than the anteroposterior annular diameter is required to ensure successful repair in the presence of a restricted posterior

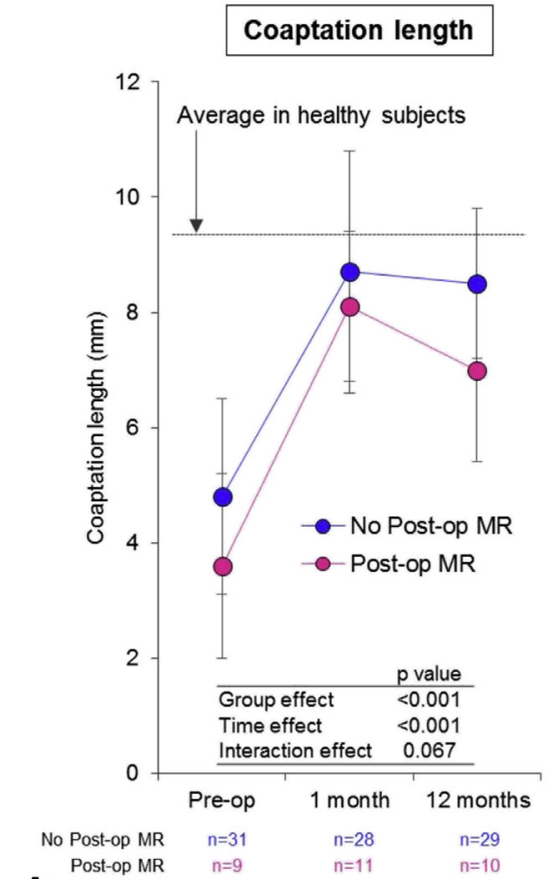

A

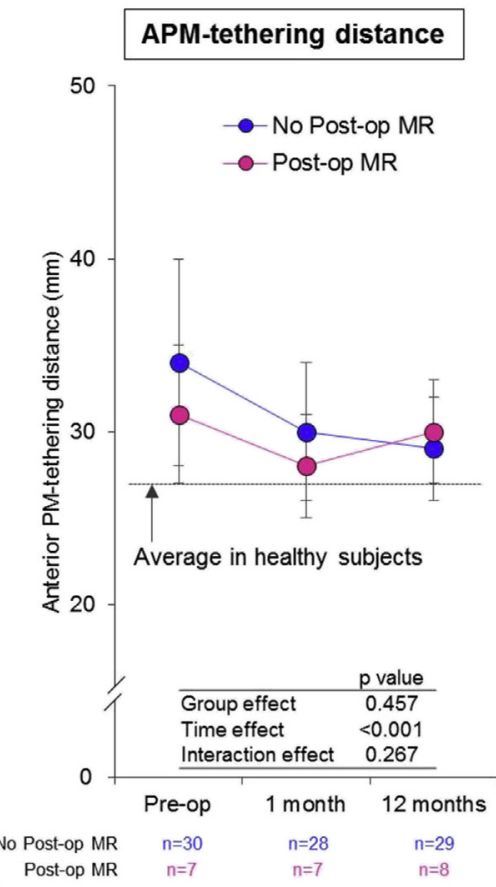

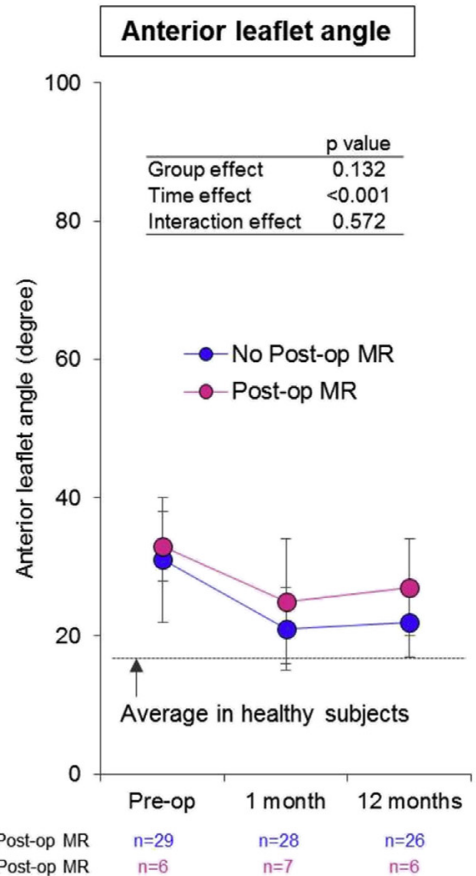

C

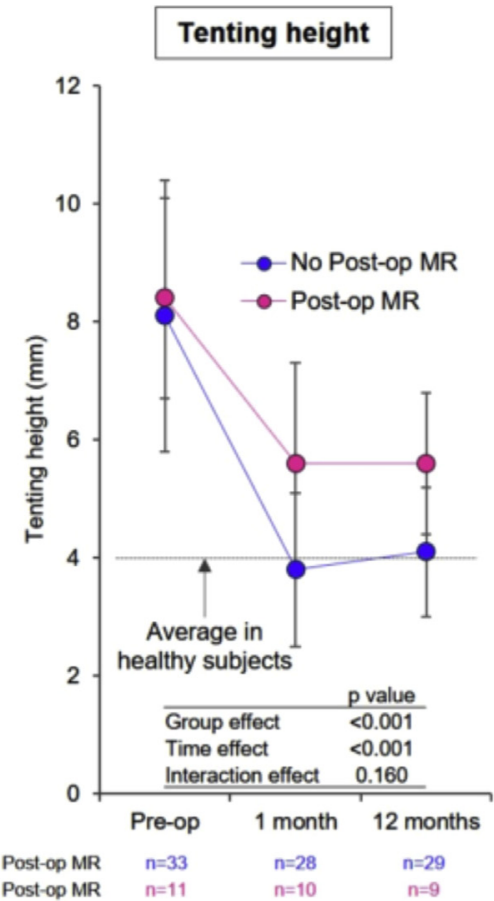

Post-op MR

FIGURE 4. Serial changes in coaptation length (A), anterior papillary muscle (APM) tethering distance (B), and anterior leaflet angle (C) according to presence and absence of mitral regurgitation $(M R)$ recurrence. Pre-op, Preoperative. 


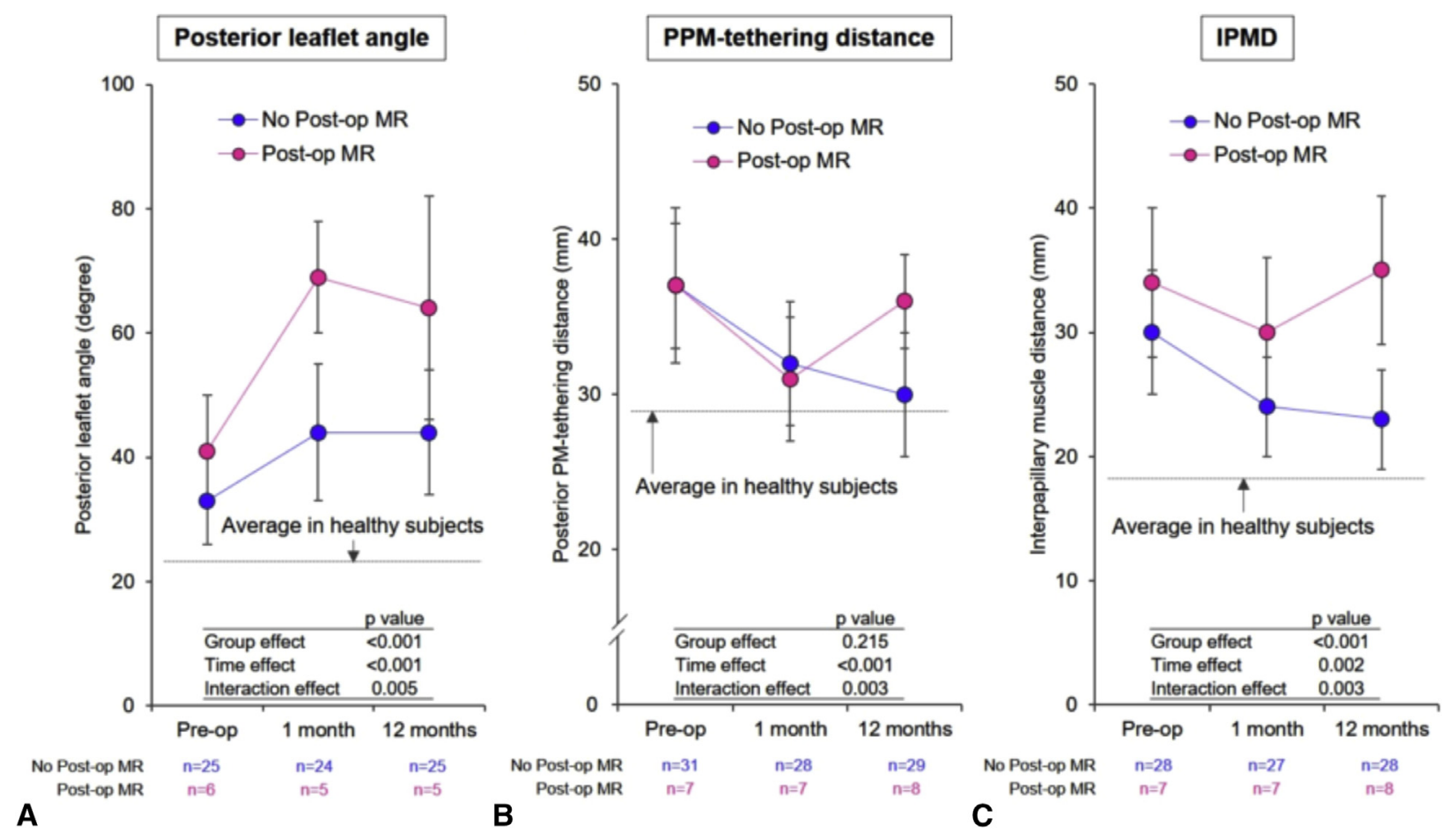

FIGURE 5. Serial changes in posterior leaflet angle (A), posterior papillary muscle $(P P M)$ tethering distance (B), and interpapillary muscle distance $(I P M D)(\mathrm{C})$ according to presence and absence of mitral regurgitation $(M R)$ recurrence. Pre-op, Preoperative.

leaflet, ${ }^{5}$ and thus aggressive undersized annuloplasty would be needed for long-term valve competence. The positive correlation between change in the annular anteroposterior diameter and that in the MR severity identified in our study supported their findings and suggests that the more aggressive reduction of the annular anteroposterior diameter, the more effective in reducing MR severity. In terms of the rate of moderate or severe recurrent MR, it was substantially lower in our study than that reported in a recent prospective randomized clinical study of $32.6 \%$ at 1 year, and $58.8 \%$ at 2 years. ${ }^{13}$ The difference in the MR recurrence rate could be explained by the differences in the baseline LV function, MR severity, degree of leaflet tethering, or the size of mitral ring implanted during repair. In a clinical study, Goldstein and colleagues reported an average of mitral annulus size of $31.0 \mathrm{~mm}$ and an average ring size of $27.9 \mathrm{~mm}$. In our study, the absolute values substantially differed, in which the annular anteroposterior diameter was $33 \pm 5 \mathrm{~mm}$ and the average ring size implanted was $25 \pm 1 \mathrm{~mm}$, indicating a greater degree of restriction applied. These findings led us to speculate that a true undersizing is a key for achieving an appropriate leaflet coaptation (valve competence) and to ensure durable results.

However, previous investigations raised the issue that reducing mitral annular size by implanting a small mitral ring shifts the posterior annulus anteriorly and potentially augments posterior leaflet tethering, potentially related to the development of postoperative recurrent MR after surgical annuloplasty. ${ }^{5-7}$ In accordance with their findings, we also confirmed that the posterior leaflet angle was increased after RMA, indicating that RMA reduces anteroposterior annular diameter at the expense of augmented tethering angle of the posterior leaflet. ${ }^{5}$ However, most importantly, our findings suggested that the RMA procedure did not increase the posterior PM tethering "distance," even in the presence of augmented posterior tethering "angle." In addition to the finding of a significant positive correlation between the changes in IPMD and LV end-systolic dimension, it was also confirmed that the change in posterior PM tethering "distance" was not correlated with corresponding change in the posterior leaflet "angle," but with changes in the LV end-systolic dimension and LV ejection fraction (data not shown). These findings lead us to consider that global LV reverse remodeling mediated by RMA might play a key role in relieving subvalvular tethering distance and offset the negative effect of augmented posterior leaflet angle induced by the restriction of the mitral annulus in selected patients. ${ }^{14}$

It remains unclear to what extent RMA alone relieves subvalvular leaflet tethering, the main mechanism of functional MR. In our echocardiographic assessments, the patients with postoperative MR recurrence showed asymmetrical tethering with displacements most prominent for the posteromedial PM, which is consistent with the 
previous findings. ${ }^{5}$ In contrast, patients without MR recurrence restored subvalvular apparatus to a greater degree with symmetrical tethering in terms of a comparable degree of anterior and posterior tethering. It is also suggested that LV remodeling is more substantially attenuated in patients whose repair is successful long-term. These findings suggest that RMA with and without surgical revascularization could benefit selected patients by reversing LV remodeling and restoring overall mitral valve geometry toward normal, thereby ensuring long-term repair durability. Taken together, earlier surgery would be warranted to provide better prognosis for patients with medically refractory functional MR, before irreversible LV remodeling occurs.

\section{Clinical Implications}

The tethering of mitral leaflets by the displaced PMs because of continued LV remodeling and/or myocardial disease progression is the main pathophysiologic mechanism of functional MR. With the fundamental mechanism of mitral leaflet tethering, RMA using a small prosthetic mitral ring can correct annular alteration, whereas it does not directly address tethering by the remodeled ventricle. Indeed, $25 \%$ of our patients failed to achieve durable repair and reverse LV remodeling. Braun and colleagues reported that it was difficult for patients with severe LV remodeling (ie, LV end-diastolic dimension $>65 \mathrm{~mm}$ or LV end-systolic dimension $>55 \mathrm{~mm}$ ) to achieve $\mathrm{LV}$ reverse remodeling by RMA alone. ${ }^{15}$ Because the mean value for preoperative LV end-diastolic dimension and LV end-systolic dimension was $67 \pm 9 \mathrm{~mm}$ and $59 \pm 9 \mathrm{~mm}$, respectively, in patients with postoperative MR, it might be reasonable for those patients to gain the limited efficacy by receiving RMA alone. Approaches that also alleviate ventricular remodeling could therefore potentially be part of a more comprehensive and effective management strategy for patients with functional MR secondary to dilated cardiomyopathy. Recently, there are some opinions in favor of adding subvalvular procedures to mitral annuloplasty to reduce the tenting forces and improve the long-term repair results. ${ }^{16,17}$ On serial quantitative assessments with multidetector computed tomography, Shudo and colleagues elegantly showed that surgical annuloplasty combined with PM approximation improved leaflet tethering to a great degree compared with annuloplasty alone, suggesting that concomitant PM approximation might be beneficial in selected patients with functional MR and advanced cardiomyopathy. ${ }^{16}$ Importantly, a significant positive correlation between change (decrease) in the tenting height and change (improvement) in the IPMD, which was observed in patients who underwent annuloplasty in addition to PM approximation might indicate that PM approximation contributes at least partly on relief of the mitral leaflet tethering. Furthermore, in a recent randomized study from Nappi and colleagues, mitral annuloplasty in addition to PM approximation was shown to exert a long-term beneficial effect on LV remodeling and restored the mitral valve geometric configuration more effectively compared with annuloplasty alone in patients with severe functional MR, consequently leading to improved long-term cardiac outcomes and repair durability. ${ }^{17}$ Finally, we found that postoperative tenting height, coaptation length, anterior leaflet angle, and posterior PM tethering distance in patients without postoperative MR were almost comparable with those of the control participants (normal subjects), indicating that the RMA procedure restored mitral valve geometry toward normal to a certain extent in selected patients, whereas the posterior leaflet angle and IPMD were not completely restored after RMA even if postoperative MR was well controlled. In addition to these observations, the independent and positive correlation between the change in MR severity and that in the IPMD might support those previous opinions in favor of using subvalvular procedures in addition to the RMA. Nonetheless, its effect on postoperative improvement in regional and global LV wall motion (LV reverse remodeling), which is responsible for repair durability, remains to be determined by a large-scale clinical research study.

\section{Limitations}

This study was retrospective in nature and investigated a small number of subjects. The inclusion of patients with ischemic and nonischemic cardiomyopathy and those who had undergone concomitant coronary artery bypass grafting might have influenced the results. However, these concomitant procedures are usually required in a population of very sick patients who present with similar clinical and pathophysiologic status despite the different etiology of LV dysfunction. To minimize potential bias related to patient selection, we excluded patients with less LV remodeling with an ejection fraction $>40 \%$ and those who underwent RMA with partial ring or concomitant surgical ventricular reconstruction. Therefore, our results would not be applicable to patients with RMA with a partial mitral ring or those with less LV remodeling.

In addition, the lengths of the anterior and posterior mitral leaflets, which were not evaluated in this study, have been shown to be useful parameters for predicting post-RMA MR recurrence. ${ }^{18}$ The assessment of these sophisticated echocardiographic parameters would have been informative to comprehensively understand the determinants of postoperative MR severity. Finally, quantitative grading of MR severity (eg, effective regurgitation orifice area and regurgitant volume) rather than the traditional semiquantitative measurement is a more objective evaluation. We believe, however, that the conclusions with respect to serial echocardiographic results are not significantly altered by such a more precise method for grading of MR severity. 


\section{CONCLUSIONS}

In patients with clinically relevant functional MR secondary to advanced cardiomyopathy, the RMA procedure partially relieved leaflet tethering, evidenced by decreased tethering distances and IPMD; the latter was the main determinant of MR. The RMA procedure did not always increase the posterior PM tethering "distance," even in the presence of augmented posterior tethering "angle." Global LV reverse remodeling mediated by RMA might play a key role in relieving subvalvular tethering and offset the negative effect of augmented posterior leaflet angle induced by the restriction of mitral annulus.

\section{Conflict of Interest Statement}

Authors have nothing to disclose with regard to commercial support.

The authors thank Mr Kiyoshi Yoshida (Section of Clinical Engineering, Japan Organization of Occupational Health and Safety, Osaka Rosai Hospital) for excellent management of the cardiopulmonary bypass procedures and Mr Naoki Kimura, Mrs Minako Furukawa, Mr Yuji Masaki, Mr Shin-ichi Fujita (Section of Clinical Echocardiography, Japan Organization of Occupational Health and Safety, Osaka Rosai Hospital) for their great assistance with echocardiographic data collection. We also thank Miss Mariko Yamashita for her great help of clinical data collection.

\section{References}

1. Kumanohoso T, Otsuji Y, Yoshifuku S, Matsukida K, Koriyama C, Kisanuki A, et al. Mechanism of higher incidence of ischemic mitral regurgitation in patients with inferior myocardial infarction: quantitative analysis of left ventricular and mitral valve geometry in 103 patients with prior myocardial infarction. $J$ Thorac Cardiovasc Surg. 2003;125:135-43.

2. Roshanali F, Mandegar MH, Yousefnia MA, Rayatzadeh H, Alaeddini F. A prospective study of predicting factors in ischemic mitral regurgitation recurrence after ring annuloplasty. Ann Thorac Surg. 2007;84:745-9.

3. Shudo Y, Matsue H, Toda K, Hata H, Fujita S, Taniguchi K, et al. A simplified echocardiographic measurements of direct effects of restrictive annuloplasty on mitral valve geometry. Echocardiography. 2010;27:931-6.

4. Green GR, Dagum P, Glasson JR, Nistal JF, Daughters GT II, Ingels NB Jr, et al. Restricted posterior leaflet motion after mitral ring annuloplasty. Ann Thorac Surg. 1999;68:2100-6.

5. Kuwahara E, Otsuji Y, Iguro Y, Ueno T, Zhu F, Mizukami N, et al. Mechanism of recurrent/persistent ischemic/functional mitral regurgitation in the chronic phase after surgical annuloplasty: importance of augmented posterior leaflet tethering Circulation. 2006;114:529-34.

6. Otsuji Y, Levine RA, Takeuchi M, Sakata R, Tei C. Mechanism of ischemic mitral regurgitation. J Cardiol. 2008;51:145-56.

7. Zhu F, Otsuji Y, Yotsumoto G, Yuasa T, Ueno T, Yu B, et al. Mechanism of persistent ischemic mitral regurgitation after annuloplasty: importance of augmented posterior mitral leaflet tethering. Circulation. 2005;112:1396-401.

8. De Bonis M, Lapenna E, Verzini A, La Canna G, Grimaldi A, Torracca L, et al. Recurrence of mitral regurgitation parallels the absence of left ventricular reverse remodeling after mitral repair in advanced dilated cardiomyopathy. Ann Thorac Surg. 2008;85:932-9.

9. Gelsomino S, Lorusso R, Capecchi I, Rostagno C, Romagnoli S, Billè G, et al. Left ventricular reverse remodeling after undersized mitral ring annuloplasty in patients with ischemic regurgitation. Ann Thorac Surg. 2008:85:1319-30

10. Hung J, Papakostas L, Tahta SA, Hardy BG, Bollen BA, Duran CM, et al. Mechanism of recurrent ischemic mitral regurgitation after annuloplasty: continued LV remodeling as a moving target. Circulation. 2004;110:85-90.

11. Kainuma S, Taniguchi K, Daimon T, Sakaguchi T, Funatsu T, Kondoh H, et al. Osaka Cardiovascular Surgery Research (OSCAR) Group. Does stringent restrictive annuloplasty for functional mitral regurgitation cause functional mitral stenosis and pulmonary hypertension? Circulation. 2011;124:97-106.

12. Kainuma S, Taniguchi K, Toda K, Funatsu T, Kondoh H, Miyagawa S, et al Osaka Cardiovascular Surgery Research (OSCAR) Group. Predictors and clinical impact of functional mitral stenosis induced by restrictive annuloplasty for ischemic and functional mitral regurgitation. Circ J. 2017;81: $1832-8$.

13. Goldstein D, Moskowitz AJ, Gelijns AC, Ailawadi G, Parides MK, Perrault LP, et al. Two-year outcomes of surgical treatment of severe ischemic mitral regurgitation. N Engl J Med. 2016:374:344-53.

14. Takeda K, Taniguchi K, Shudo Y, Kainuma S, Hamada S, Matsue H, et al. Mechanism of beneficial effects of restrictive mitral annuloplasty in patients with dilated cardiomyopathy and functional mitral regurgitation. Circulation. 2010; 122:S3-9.

15. Braun J, van de Veire NR, Klautz RJ, Versteegh MI, Holman ER, Westenberg JJ, et al. Restrictive mitral annuloplasty cures ischemic mitral regurgitation and heart failure. Ann Thorac Surg. 2008;85:430-7.

16. Shudo Y, Matsumiya G, Sakaguchi T, Miyagawa S, Yoshikawa Y, Yamauchi T, et al. Assessment of changes in mitral valve configuration with multidetector computed tomography: impact of papillary muscle imbrication and ring annuloplasty. Circulation. 2010;122:S29-36.

17. Nappi F, Lusini M, Spadaccio C, Nenna A, Covino E, Acar C, et al. Papillary muscle approximation versus restrictive annuloplasty alone for severe ischemic mitral regurgitation. J Am Coll Cardiol. 2016;67:2334-46.

18. Yoshida S, Fukushima S, Miyagawa S, Nakamura T, Yoshikawa Y, Hata H, et al Mitral valve structure in addition to myocardial viability determines the outcome of functional mitral regurgitation after coronary artery bypass grafting. Circ J. 2017;81:1620-7.

Key Words: restrictive mitral annuloplasty, functional mitral regurgitation, leaflet tethering, interpapillary muscle distance 
TABLE E1. Determinants of $\Delta$ MR severity

\begin{tabular}{|c|c|c|c|c|}
\hline & Parameter estimate & Standard error & $t$ statistic & $P$ value \\
\hline \multicolumn{5}{|l|}{ Patient demographic characteristic } \\
\hline Male sex & 0.657 & 0.395 & 1.664 & .114 \\
\hline \multicolumn{5}{|l|}{ LV function parameters } \\
\hline$\Delta$ LVESD, $\mathrm{mm}$ & 0.038 & 0.022 & 1.725 & .102 \\
\hline \multicolumn{5}{|l|}{ Subvalvular configuration } \\
\hline$\Delta$ APM-tethering distance, $\mathrm{mm}$ & 0.050 & 0.047 & 1.069 & .299 \\
\hline$\Delta \mathrm{PPM}$-tethering distance, $\mathrm{mm}$ & -0.104 & 0.045 & -2.281 & .035 \\
\hline$\Delta$ Posterior leaflet angle, degree & -0.024 & 0.013 & -1.893 & .075 \\
\hline$\Delta \mathrm{IPMD}, \mathrm{mm}$ & 0.100 & 0.039 & 2.584 & .019 \\
\hline
\end{tabular}

$\overline{L V}$, Left ventricle; $\triangle$, change; $L V E S D$, left ventricular end-systolic dimension; $A P M$, anterior papillary muscle; $P P M$, posterior papillary muscle; $I P M D$, interpapillary muscle distance.

TABLE E2. Determinants of $\Delta$ interpapillary muscle distance

\begin{tabular}{|c|c|c|c|c|}
\hline Variables & Parameter estimate & Standard error & $t$ statistic & $P$ value \\
\hline \multicolumn{5}{|l|}{ Patient demographic characteristic } \\
\hline Male sex & -0.330 & 2.205 & -1.495 & .151 \\
\hline \multicolumn{5}{|l|}{ LV function parameters } \\
\hline$\Delta$ LVESD, mm & 0.299 & 0.110 & 2.727 & .013 \\
\hline \multicolumn{5}{|l|}{ Subvalvular configuration } \\
\hline$\Delta$ APM tethering distance, $\mathrm{mm}$ & -0.290 & 0.268 & -1.084 & .292 \\
\hline$\Delta$ PPM tethering distance, $\mathrm{mm}$ & 0.348 & 0.256 & 1.360 & .190 \\
\hline$\Delta$ Posterior leaflet angle, degree & 0.127 & 0.068 & 1.868 & .077 \\
\hline
\end{tabular}

$L V$, Left ventricle; $\triangle$, change; $L V E S D$, left ventricular end-systolic dimension; $A P M$, anterior papillary muscle; $P P M$, posterior papillary muscle. 